\title{
A Novel Phenylimidazo[1,2-a]pyridine Core Skeleton and Pharmacophore Model as Hypoxia-Inducible Factor Inhibitors
}

\author{
Young-Dae Gong* and Nam Sook Kang ${ }^{\dagger}{ }^{* *}$ \\ Innovative Drug Library Research Center, Department of Chemistry, Dongguk University-Seoul, \\ Seoul 100-715, Korea.*E-mail:ydgong@dongguk.edu \\ ${ }^{\dagger}$ Center for Drug Discovery Platform Technology, Korea Research Institute of Chemical Technology, P.O. Box 107, \\ Daejeon 305-600, Korea. "E-mail: nskang@krict.re.kr \\ Received March 15, 2010, Accepted May 3, 2010
}

Key Words: Phenylimidazo[1,2-a]pyridine, Hypoxia-inducible factor (HIF), Drug-like core skeleton, Pharmacophore

Hypoxia-inducible factor (HIF) is a transcription factor induced by hypoxia and degraded by ubiquitin-dependent proteasomes in normoxic conditions. HIF- $1 \alpha$ regulates the transcription of many genes involved in key aspects of cancer biology, including various immortalizations, maintenance of stem cell pools, cellular dedifferentiation, genetic instability, vascularization, metabolic reprogramming, autocrine growth factor signaling, invasion/metastasis, and treatment failure. ${ }^{1}$ Hypoxic tumor cells are resistant to conventional chemotherapy and radio-therapy. ${ }^{2-4}$ Consequently, the presence of hypoxia in tumors plays a negative role in patient prognosis.

The importance of HIF- $1 \alpha$ in the mediation of normal and pathological processes has motivated considerable efforts to identify HIF- $1 \alpha$ inhibitors. Although a wealth of inhibitory compounds are available, synthesizing HIF-1 $\alpha$ inhibitors with a novel small-molecule core skeleton fitting individual HIF- $1 \alpha$

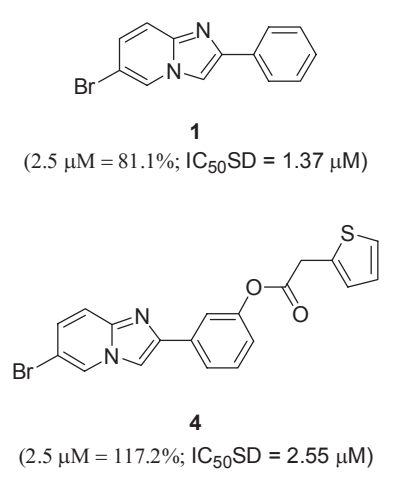

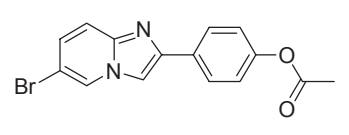

2

$$
\left(2.5 \mu \mathrm{M}=110.3 \% ; \mathrm{IC}_{50} \mathrm{SD}=0.14 \mu \mathrm{M}\right)
$$

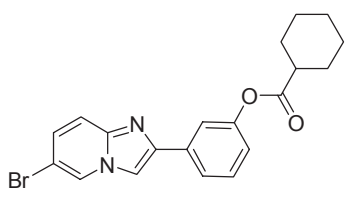

5

$(2.5 \mu \mathrm{M}=53.9 \%)$

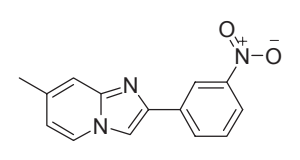

$\left(2.5 \mu \mathrm{M}=71.5 \% ; \mathrm{IC}_{50} \mathrm{SD}=\mathrm{NA}\right.$ up to $\left.2.5 \mu \mathrm{M}\right)$

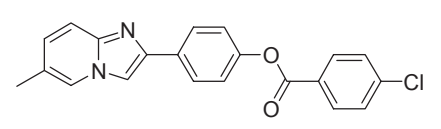

11

$\left(\mathrm{IC}_{50} \mathrm{SD}>1\right)$

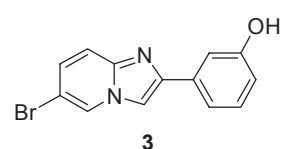

$\left(2.5 \mu \mathrm{M}=126.7 \% ; \mathrm{IC}_{50} \mathrm{SD}=0.41 \mu \mathrm{M}\right)$

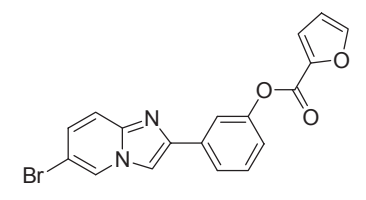

6

$\left(2.5 \mu \mathrm{M}=95.9 \% ; \mathrm{IC}_{50} \mathrm{SD}=0.26 \mu \mathrm{M}\right)$

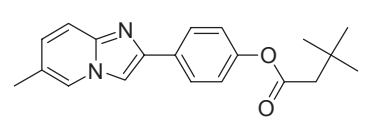

9

$\left(\mathrm{IC}_{50} \mathrm{SD}>1\right)$

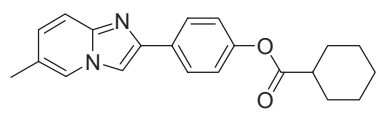

12

$\left(\mathrm{IC}_{50} \mathrm{SD}>1\right)$

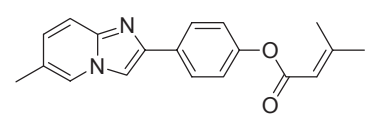

13

$\left(\mathrm{IC} \mathrm{C}_{50} \mathrm{SD}>1\right)$

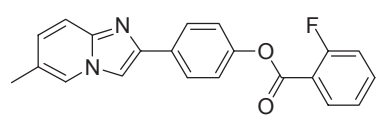

14

$\left(\mathrm{IC} \mathrm{C}_{50} \mathrm{SD}>1\right)$

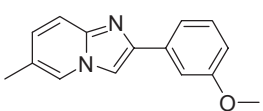

15

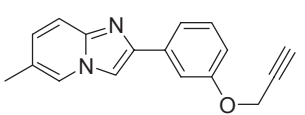

16 $\left(\mathrm{IC} \mathrm{C}_{50} \mathrm{SD}>1\right)$

Figure 1. Structure of HIF-1 $\alpha$ inhibitors (1-8) based on phenylimidazo[1,2- $a$ ]pyridine core skeleton identified in primary screen. Structure of the compounds with no or little HIF-1 $\alpha$ inhibitory activity (9-16). 


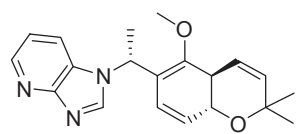

R1

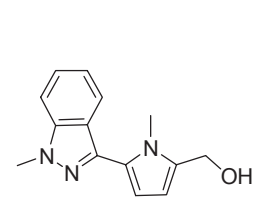

R3

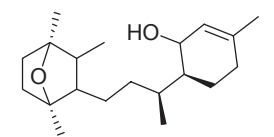

R2

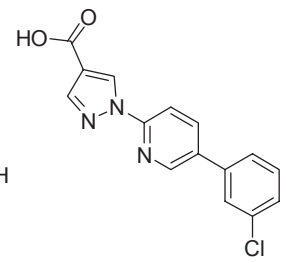

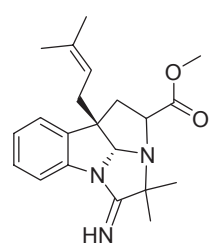

R5
Figure 2. The reference compounds in the training set obtained from prous.integrity.com site as HIF- $1 \alpha$ inhibitors for the pharmacorphore model.

has proven to be a challenge. Many reports exist describing the development of anticancer agents targeting HIF-1 $\alpha$, but only some anticancer agents, incorporating heterocyclic rings and several aromatic core skeletons, have been identified as inhibitors of HIF- $1 \alpha$ activity. ${ }^{5-6}$ We therefore undertook an investigation aimed at developing novel and simple drug-like heterocyclic derivatives as inhibitors of HIF-1 $\alpha .^{7}$ By using the bioisostere concept based on benzene ring fused bicyclic HIF-1 $\alpha$ inhibitors, we have designed scaffolds that combine the pyridine moiety found in phenylimidazo[1,2-a]pyridine ring system.

Herein, we report initial evidence showing that compounds with a phenylimidazo[1,2- $a$ ]pyridine core skeleton are selective HIF-1 $\alpha$ inhibitors, and have potential as anticancer agents in treatment. The aim of this study was to investigate the biochemical properties of HIF-1 $\alpha$ and to identify new core skeleton inhibitors of HIF-1 $\alpha$. To identify small-molecule inhibitors of HIF-1 $\alpha$, we screened 1,200 compounds using the HRE/ SW480 stable cell line containing HRE-luc[pHTS-9XHRELuc]. Once HIF-1 $\alpha$ was induced, the HRE (hypoxia response element) promoter was activated by binding with $\mathrm{HIF}-1 \alpha$, which turns on the reporter gene, luciferase. Therefore, the compounds can be tested for whether HIF-1 $\alpha$ activation is inhibited using this system. These 1,200 compounds represent structurally diverse and druggable 20,000-member heterocyclic compound libraries, including imidazole, benzopyrans, oxazoles, thiazoles, thiadiazoles, and pyridines. ${ }^{8-12}$

The primary screen identified several phenylimidazo[1,2- $a$ ] pyridines (Figure 1, 1-8 compounds) as inhibitors of HIF-1 $\alpha$ cell activities (Figure 1). Despite their structural similarity to the phenylimidazo[1,2-a]pyridine scaffold (Figure 1, 9-16 compounds) from the library showed little or no HIF-1 $\alpha$ inhibitory activity in the primary screen. From preliminary structure-activity relationship studies of 1-16, it appears that the phenylimidazo[1,2-a]pyridine moiety is critical to their HIF-1 $\alpha$ inhibitory activities, as shown in Figure 1.

To clearly investigate the structure-activity relationship studies of 1-16, we selected known 67 compounds from the Prous site (http://prous.integrity.com) as HIF-1 $\alpha$ inhibitors. After 3D structures of these compounds were generated using CONCORD, ${ }^{13}$ they were minimized using Gasteiger-Huckel

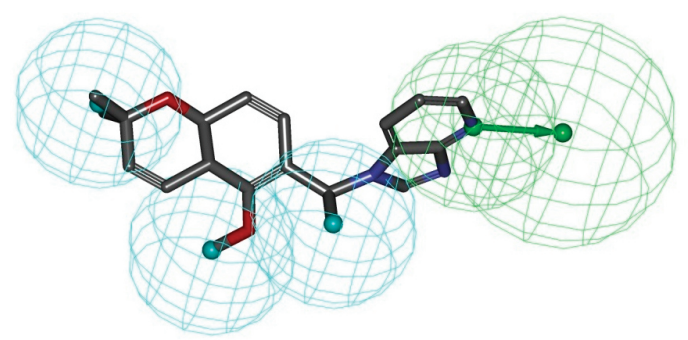

Figure 3. Pharmacophore mapping for reference compound R1, having Fitvalue 4.0. The green sphere represents the hydrogen-bond acceptor site, cyan the hydrophobic sites. All compounds in the training set above have a Fitvalue of 2.9 or more.

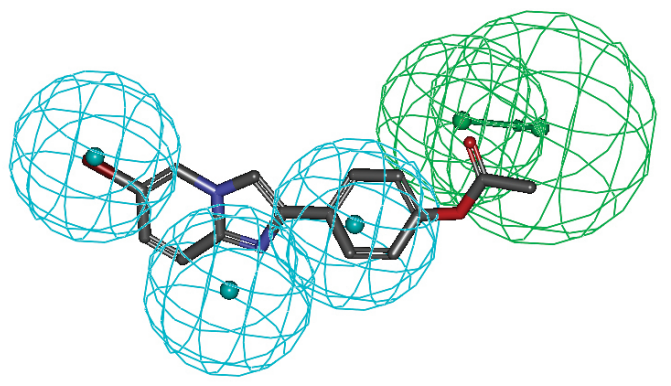

Figure 4. Pharmacophore mapping for compound 2, having a Fitvalue 2.90 .

charges and conjugated gradient methods. In order to consider the structural redundancy of the subset, we carried out the maximum dissimilarity study using the Extended Connectivity Fingerprints (ECFP). ${ }^{14}$ We selected 5 reference compounds as the training set, as shown in Figure 2. Based on the bioisostere concept, we considered the selected references (especially Figure 2, R1- $\mathbf{R 5}^{15-19}$ ) as having quite similar core skeletons with our desired phenylimidazo[1,2-a]pyridine structure. Common feature pharmacophores are generated using the HipHop algorithm. ${ }^{20}$ Predefined pharmacophore features are used to automatically create the pharmacophores hypothesis model. The list of features of minimum and maximum values was as follows: $\mathrm{H}$-bond acceptor (Hba) 0 and 5, H-bond donor (Hbd) 0 and 5, Hydrophobic (Hy) 0 and 5. The compounds in the training set showed fit values ranging from 2.9 to 4.0. Our compounds showed a fit value range from 0.3 to 3.2 .

The pharmacophore mapping for reference compound R1 having Fitvalue 4.0 is shown in Figure 3. The green sphere represents the hydrogen-bond acceptor site, and cyan the hydrophobic sites. All compounds in the training set had a Fitvalue of 2.9 or more. Our efforts were particularly focused on to calculating pharmacophore mapping of the structure of $\mathbf{R} \mathbf{1}$, since the conformation structures of our hit compounds had similar R1's. The pharmacophore mapping for compounds $\mathbf{2}$ and $\mathbf{6}$, which present the highest activity, having fit values of 2.90 and 3.23, respectively, are also shown in Figures 4 and 5. The oxygen atom on the ester group of compounds $\mathbf{2}$ and $\mathbf{6}$ have hydrogen-bond acceptor roles like the nitrogen atom on the pyridine ring of $\mathbf{R} \mathbf{1}$, as shown in Figures 3 and 4 (green sphere represents the hydrogen-bond acceptor site). However, 


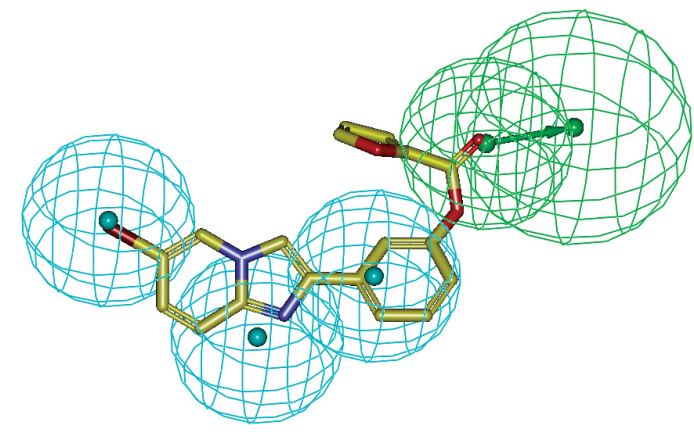

Figure 5. Pharmacophore mapping for Compound 6, having a Fitvalue of 3.23 .

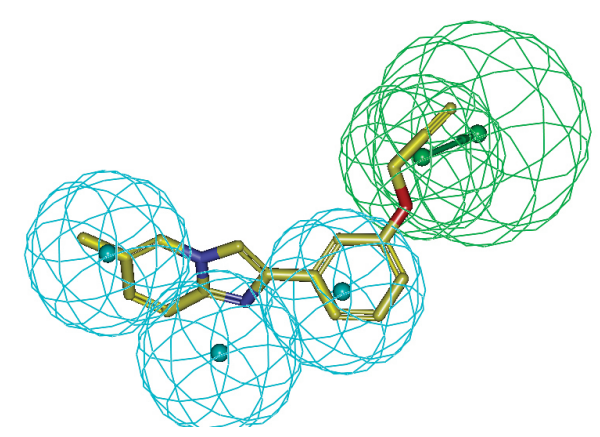

Figure 6. Pharmacophore mapping for Compound 16, having a fitvalue of 0.38 . This compound did not match the hydrogen-bond acceptor site, resulting in decreased activity.

Table 1. The fit values for 16 compounds shown in Figure 1

\begin{tabular}{ccc}
\hline Compounds & \% inhibition at $2.5 \mathrm{uM}$ & Fitvalue \\
\hline 1 & 81.1 & 1.00 \\
2 & 110.3 & 2.90 \\
3 & 126.7 & 2.73 \\
4 & 117.2 & 2.93 \\
5 & 53.9 & 2.93 \\
6 & 95.9 & 3.23 \\
7 & 2.2 & 2.98 \\
8 & 71.5 & 1.98 \\
9 & $-a$ & 1.99 \\
10 & - & 1.95 \\
11 & - & 2.74 \\
12 & - & 2.91 \\
13 & - & 2.09 \\
14 & - & 1.98 \\
15 & - & 2.60 \\
16 & - & 0.38 \\
\hline
\end{tabular}

${ }^{a}$ Compounds with no or little HIF-1 $\alpha$ inhibitory activity.

when the ether group instead of the ester group was introduced on the benzene ring, compound $\mathbf{1 6}$ did not match the hydrogenbond acceptor site, and presented a very low fitvalue of 0.38 , resulting in decreased activity. The fit values of the other compounds shown in Figure 1 are listed in Table 1.

In summary, we screened 1,200 heterocyclic small molecules, and identified a novel hit core skeleton of phenylimidazo[1,2- $a]$ pyridine, selected as a potential HIF-1 inhibitor and demonstrated its effects in inhibiting HIF-1 $\alpha$, leading to inhibition of hypoxia-induced responses in vitro and pharmacophore analysis. Further studies are currently underway to optimize the potency and selectivity of phenylimidazo[1,2- $a]$ pyridine acetates (Figure 1, compound 2-6) and address their in vivo efficacy and therapeutic potential. These molecules may serve as useful mechanistic probes of the cellular function of HIF-1 $\alpha$ and the anticancer potential of biological mechanisms underlying compulsive chronic cancer agents.

\section{Experimental Procedure}

General for synthesis. All chemicals were reagent grade and used as purchased. Reactions were monitored by TLC analysis using Merck silica gel 60 F-254 thin layer plates or ATR-FTIR analysis using TravelIR ${ }^{\mathrm{TM}}$ (SensIR Technology). Flash column chromatography was carried out on Merck silica gel 60 (230 - 400 mesh). On solid-phase synthesis, reactions, filtration, and washing were carried out on a MiniBlock (Bohdan) and solvent evaporation was performed on a GeneVac Atlas HT-4 centrifugal vacuum evaporator. The crude products were purified by parallel chromatography using Quad3 ${ }^{\mathrm{TM}} \cdot{ }^{1} \mathrm{H}$ NMR and ${ }^{13} \mathrm{C}$ NMR spectra were recorded in $\delta$ units relative to deuterated solvent as an internal reference using a Bruker $500 \mathrm{MHz}$ NMR instrument. LC-MS analysis was performed on an ESI mass spectrometer with PDA detection. LC-MS area \% purities of all products were determined by LC peak area analysis (XTerraMS $\mathrm{C}_{18}$ column, $4.6 \mathrm{~mm} \times 100 \mathrm{~mm}$; PDA detector at $200-400 \mathrm{~nm}$; gradient, $\left.5-95 \% \mathrm{CH}_{3} \mathrm{CN} / \mathrm{H}_{2} \mathrm{O}\right)$.

Representative synthesis of 2-\{4-(2-fluorobenzyoxy ester) phenyl\} -6-methyl imidazo[1,2-a] pyridine (14): We prepared 2-(4-hoxyphenyl)-6-methylimidazo[1,2- $a$ ]pyridine as a precursor of the final products by a previously-reported method. ${ }^{21}$ Then, to a mixture of 2-(4-hoxyphenyl)-6-methylimidazo $[1,2-a]$ pyridine $(40.0 \mathrm{mg}, 0.179 \mathrm{mM})$ in the mixture of tetrahydofuran (THF) and $N, N$-dimethylformamide (DMF) (4:1, $3 \mathrm{~mL}$ ) solution at room temperature was added 2-fluoro-benzoyl chloride ( $33.9 \mathrm{mg}, 0.214 \mathrm{mM}, 1.2$ equiv.) and triethylamine (27.1 mg, $0.268 \mathrm{mM}, 1.5$ equiv.) with 4- $N, N$-dimethylamino pyridine (DMAP, cat. 0.1 equiv.), and the mixture was heated at $50{ }^{\circ} \mathrm{C}$ for $3 \mathrm{~h}$. Evaporation of the solvent gave a residue which was purified by column chromatograph [ethylacetate/ hexane $1 / 4(\mathrm{v} / \mathrm{v})$ as eluent], and yielded the desired product $14(32 \mathrm{mg}$, 51\%). ${ }^{1} \mathrm{H}$ NMR (500 MHz, $\left.\mathrm{CDCl}_{3}\right) \delta 8.15-8.12$ (m, 1H), 8.00$7.98(\mathrm{~m}, 2 \mathrm{H}), 7.89(\mathrm{~s}, 1 \mathrm{H}), 7.76(\mathrm{~s}, 1 \mathrm{H}), 7.65-7.59(\mathrm{~m}, 1 \mathrm{H})$, $7.53(\mathrm{~d}, 1 \mathrm{H}, J=9.2 \mathrm{~Hz}), 7.31-7.29(\mathrm{~m}, 3 \mathrm{H}), 7.28-7.26(\mathrm{~m}, 1 \mathrm{H})$, 7.03-7.02 (m, 1H). LC/MS (ESI) $m / z 347\left([\mathrm{M}+\mathrm{H}]^{+}\right)$.

Materials for screening. Cell culture medium, fetal bovine serum (FBS), and horse serum were obtained from Invitrogen (Gaithersburg, MD, USA). An 18-mer peptide [DLDLEALAPYIPADDFQ] as the substrate of the PHD2 enzyme, was synthesized by AnyGen Co. Ltd. (Kwangju, Korea). The 18-mer peptide matches the amino acid residues 556 to 575 of HIF- $1 \alpha$. To identify PHD activators, chemical compounds were obtained from Korea Research Institute of Chemical Technology (Daejeon, Korea). All of the other chemicals were purchased from Sigma (St. Louis, MO, USA) and/or the same as described ${ }^{7}$ 
unless otherwise indicated.

Inhibitory efficacy test of the compounds on cancer cell proliferation. To evaluate the anti-cancer effect of the synthetic compounds, the colon cancer cell line HCT116 was used to test the inhibitory effect on cancer cell proliferation. HCT116 was cultured with DMED containing $10 \% \mathrm{FBS}$ at $37{ }^{\circ} \mathrm{C}$.

Cell culture. HCT 116 cells cultured in T75 were transferred to 96 well plates as follows. When cells reached a confluence, the cells were trypsinized using trypsin-EDTA solution for 5 minutes after washing with PBS. Subsequently, $10 \mathrm{~mL}$ of culture media was added and the mixture was centrifuged at 1,500 rpm for 3 minutes. The supernatant was aspirated out and the cell pellet was resolved in $10 \mathrm{~mL}$ of culture media. The cells were counted and transferred to a 96-well plate as 5,000 cells/ $100 \mu \mathrm{L} /$ well. The cells were incubated for 24 hours in a $\mathrm{CO}_{2}$ incubator

Preparation of the testing material. The testing material stock plate was prepared as a concentration of $0.1,0.3,1,3,10$, and $30 \mathrm{mM}$ in DMSO. $2 \mu \mathrm{L}$ of stock solution was diluted in $248 \mu \mathrm{L}$ of culture media as a 1/125 dilution. The cell culture media was aspirated out, and $175 \mu \mathrm{L}$ of fresh media was added. $25 \mu \mathrm{L}$ of the prepared stock solution was added to the media, which gave concentrations of $0.1,0.3,1,3,10$, and $30 \mu \mathrm{M}$. The treated cells were incubated for 72 hours

Measurement. After 72 hours of incubation, $20 \mu \mathrm{L}$ of MTS solution (CellTiter 96 Aqueous one solution cell proliferation assay) was added and incubated for 1 hour. The reaction was measured using a Thermo-MAX microplate reader (Molecular Devices) at $490 \mathrm{~nm}$.

Inhibitory efficacy test of compounds on HIF binding using reporter assay system.

Principle of experiment: Hypoxic conditions were induced to HIF-1a using an HRE/SW480 stable cell line containing HRE-luc[pHTS-9XHRE-Luc]. 1,22 Once HIF-1a was induced, the HRE (hypoxia response element) promoter was activated by binding with HIF-1 $\alpha$, which turns on the reporter gene, luciferase. In this way, the compounds could be tested for whether HIF-1a activation is inhibited.

Experimental method: To achieve hypoxic conditions, 100 $\mu \mathrm{M}$ of $\mathrm{CoCl}_{2}$ was treated on a [pHTS-9XHRE-Luc] SW480 cell line for 24 hours. After compound treatment with a series of concentrations for 20 hours, luciferase activity was measured using Bright-glo(promega).

Computational method: We selected 67 compounds from prous.integrity.com site as HIF-1 inhibitors. After 3D structures of these compounds were generated using CONCORD, ${ }^{13}$ they were minimized using Gasteiger-Huckel charges and conjugated gradient methods. In order to consider the structural redundancy of the subset, we carried out a maximum dissimilarity study using Extended Connectivity Fingerprints (ECFP). ${ }^{14} \mathrm{We}$ selected 5 compounds as the training set, as shown in Figure 1. Common feature pharmacophores were generated using the
HipHop algorithm. ${ }^{20}$ The predefined pharmacophore features were used to automatically create the pharmacophores hypothesis model. The lists of feature of minimum and maximum values are as follows: $\mathrm{H}$-bond acceptor (Hba) 0 and 5, H-bond donor (Hbd) 0 and 5, Hydrophobic (Hy) 0 and 5.

Acknowledgments. This research was supported by a grant from the Basic Research Program of Dongguk University-Seoul, Korea.

\section{References}

1. Semenza, G. L. Drug Discov. Today 2007, 12, 853.

2. Jiang, B. H.; Rue, E.; Wang, R. Roe.; Semenza. G. L. J. Biol. Chem. 1996, 271, 17771 .

3. Wang, G. L.; Semenza, G. L. J. Biol. Chem. 1995, 270, 1230.

4. Kaelin, W. G. Annu. Rev. Biochem. 2005, 74, 115.

5. Lee, K.; Lee, J. H.; Boovanahalil, S. K.; Jin, Y.; Lee, M.; Jin, X.; Kim, J. H.; Hong, Y.-S.; Lee, J. J. J. Med. Chem. 2007, 50, 1675.

6. Giaccia, A.; Siim, B. G.; Johnson, R. S. Nat. Rev. Drug Discovery 2003, 2,1 .

7. Choi, H. J.; Song, B.-J.; Gong, Y.-D.; Gwak, W. J.; Soh, Y. British J. Pharm. 2008, 154, 114.

8. Hwang, J. Y.; Choi, H.-S.; Seo, J.-S.; La, H.-J.; Yoo, S.-e.; Gong, Y. D. J. Comb. Chem. 2006, 8, 897.

9. Hwang, J. Y.; Gong, Y. D. J. Comb. Chem. 2006, 8, 297.

10. Hwang, J. Y.; Choi, H. S.; Lee, D. H.; Yoo, S.-e.; Gong, Y. D. J. Comb. Chem. 2005, 7, 136.

11. Hwang, J. Y.; Choi, H. S.; Lee, D. H.; Gong, Y. D. J. Comb. Chem. 2005, 7, 816 .

12. Lee, I. Y.; Kim, S. Y.; Lee, J. Y.; Yu, C.-M.; Lee, D. H.; Gong, Y. D. Tetrahedron Lett. 2004, 45, 9319.

13. Concord; Tripos Inc., 1699 South Hanley Road, St. Louis, MO 63144.

14. Hert, J.; Willett, P.; Wilton, D. J.; Acklin, P.; Azzaoui, K.; Jacoby, E.; Schuffenhauer, A. J. Chem. Inf. Comp. Sci. 2004, 44, 1177.

15. Chalet, T.; Rita, G. N.; Anthony, J. R.; Beata, P.; Fatima, K.; Zhaobin, Z.; Huanchun, Z.; Quincy, T.; Ainsley, C. N.; Paraskevi, G.; Wei, Z.; Jeffrey, J. O.; Manuela, M. P.; Nicolaou, K. C.; Erwin, G. V. M. Cancer Res. 2005, 65, 605.

16. Amar, G. C.; Gundluru, M. K.; Kumar, P. B. C.; Yang, L.; YuDong, Z.; Dale, G. N.; Mitchell A. A. J. Med. Chem. 2007, 50, 6299.

17. Park, J. W.; Chun, Y. S.; Kenneth, B.; Sho, H. S. WO 2005030121. 2005.

18. Warshakoon, N. C.; Wu, S.; Boyer, A.; Kawamoto, R.; Renock, S.; Xu, K.; Pokross, M.; Evdokimov, A. G.; Zhou, S.; Winter, C.; Walter, R.; Mekel, M. Bioorg. Med. Chem. Lett. 2006, 16, 5687.

19. Ventosa-Andres, P.; Gonzalez-Vera, J. A.; Valdivielso, A. M.; Teresa Garcia-Lopez, M.; Herranz, R. Bioorg. Med. Chem. 2008, 16, 9313.

20. Sprague, P. W. Automated Chemical Hypothesis Generation and Database Searching with Catalyst. In Perspectives in Drug Discovery and Design; Müller, K., Ed.; ESCOM Science Publishers B. V.: Leiden, The Netherlands, 1995; Vol. 3, pp 1-20.

21. Leopoldo, M.; Lacivita, E.; Passafiume, E.; Contino, M.; Colabufo, N.; Berardi, F.; Perrone, Liu, R. J. Med. Chem. 2007, 50, 5043.

22. Han, Z. B.; Ren, H.; Zhao, H.; Chi, Y.; Chen, K.; Zhou, B.; Liu, Y. J.; Zhang, L.; Xu, B.; Liu, B.; Yang, R.; Han, Z. C. Carcinogenesis 2008, 29, 1853. 